\title{
Aquecimento global: efeitos no crescimento, no desenvolvimento e na produtividade de batata
}

\author{
Global warming: effects on growth, development, and yield of potato \\ Joelma Dutra Fagundes ${ }^{\text {I }}$ Gizelli Moiano de PaulaII Isabel Lago ${ }^{I I}$ \\ Nereu Augusto Streck ${ }^{\text {III* }}$ Dilson Antônio Bisognin ${ }^{\text {III }}$
}

\section{-REVISÃO BIBLIOGRÁFICA-}

\section{RESUMO}

A concentração atmosférica dos gases do efeito estufa, principalmente o $\mathrm{CO}_{2}$, tem aumentado nas últimas décadas devido às atividades antrópicas. A concentração de $\mathrm{CO}_{2}$ aumentou de aproximadamente 280 partes por milhão por volume (ppmv) no período pré-industrial para a atual concentração de 380 ppmv. Há registros que, durante o século $X X$, houve um aumento da temperatura média da superfície global de $0,6 \pm(0,2)^{\circ} \mathrm{C}$, e projeções indicam um provável aumento de 1,1 a $6,4^{\circ} \mathrm{C}$ na temperatura média global até o final do século XXI, dependendo da região do planeta. $O$ aumento da concentração de $\mathrm{CO}_{2}$ e da temperatura afeta diretamente processos fisiológicos, como fotossíntese e respiração das plantas, o que poderá alterar o desempenho das culturas, incluindo a batata. O objetivo desta revisão foi reunir informações da literatura sobre os possíveis efeitos do aumento na concentração de $\mathrm{CO}_{2}$ e da temperatura do ar no crescimento, no desenvolvimento e na produtividade da cultura de batata. O aumento do $\mathrm{CO}_{2}$ seguido de aumento na temperatura do ar, de maneira geral, resultará em menor crescimento, redução na duração do ciclo de desenvolvimento, menor produtividade e aumento da incidência de doenças da batata. Como estratégia para minimizar os efeitos de um possível aquecimento global sobre essa cultura, sugere-se que sejam desenvolvidas cultivares tolerantes a altas temperaturas, adaptadas as épocas de plantio em cada local, alteradas as práticas de manejo da cultura e até expandidas as áreas de cultivo para regiões mais frias.

Palavras-chave: Solanum tuberosum L., dióxido de carbono, aumento de temperatura, mudança climática, tratos culturais.

\section{ABSTRACT}

The concentration of atmospheric greenhouse gases, mainly the $\mathrm{CO}_{2}$, has increased in the last decades due to anthropogenic activities. The atmospheric $\mathrm{CO}_{2}$ concentration has increased from about 280 parts per million per volume (ppmv) in the pre-industrial period to the currently 380ppmv concentration. There are reports that during the $X X$ century global average temperature increased $0.6 \pm(0.2)^{\circ} \mathrm{C}$ and projections indicate a possible 1.1 to $6.4^{\circ} \mathrm{C}$ increase in temperature by the end of the XXI century, depending upon each region. The increase in atmospheric $\mathrm{CO}_{2}$ concentration and air temperature directly affect plant physiological processes, such as photosynthesis and respiration, which may affect crops performance, including potato. The objective of this review was to assemble information from the literature on the possible effects of increasing atmospheric $\mathrm{CO}_{2}$ concentration and air temperature on growth, development and yield of potato. In general, the increase in $\mathrm{CO}_{2}$ concentration followed by an increase in air temperature will result in lower growth, reduction in the duration of the developmental cycle, lower yield and increase of potato diseases. A strategy to minimize the effects of a possible global warming on potato would be to develop cultivars that are tolerant to high temperatures, adapt planting time in each location, change management practices and even, expand growing areas to colder regions.

Key words: Solanum tuberosum L., carbon dioxide, temperature increase, climate change, crop management.

IPrograma de Pós-graduação em Agronomia, Universidade Federal de Santa Maria (UFSM), Santa Maria, RS, Brasil.

"Programa de Pós-graduação em Engenharia Agrícola, UFSM, Santa Maria, RS, Brasil.

IIIDepartamento de Fitotecnia, Centro de Ciências Rurais (CCR), UFSM. Av. Roraima, 1000, 97105-900, Santa Maria, RS, Brasil.

E-mail: nstreck2@yahoo.com.br. *Autor para correspondência. 


\section{INTRODUÇÃO}

A batata (Solanum tuberosum L.), uma planta $\mathrm{C}_{3}$, é cultivada em mais de 120 países, ocupando o quarto lugar em produção de alimentos, com 314 milhões de toneladas anuais (FAOSTAT, 2009). É um dos principais alimentos consumidos pela humanidade, dada a composição nutricional, a versatilidade gastronômica e o baixo preço de comercialização dos tubérculos (ABBA, 2009a). O ano de 2008 foi declarado o ano internacional da batata pela FAO, com o objetivo de criar uma consciência sobre sua importância na luta contra a fome e a pobreza mundial. Assim, trabalhos que visam a entender como essa cultura será afetada pelo aquecimento global são de grande relevância.

O dióxido de carbono, metano, óxido nitroso e vapor d’água são os principais gases que absorvem radiação eletromagnética emitida pelos corpos terrestres, causando o fenômeno natural chamado de efeito estufa, e a concentração desses gases na atmosfera terrestre tem aumentado nas últimas décadas, em razão das atividades antrópicas (IPCC, 2007). A concentração de dióxido de carbono $\left(\mathrm{CO}_{2}\right)$ aumentou de aproximadamente 280 partes por milhão por volume (ppmv) no período pré-industrial para a atual concentração de 380 ppmv, com uma taxa de aumento de 1,6ppmv por ano (MEARNS, 2000; IPCC, 2007). Há registros de que, durante o século $\mathrm{XX}$, houve um aumento da temperatura média da superfície global de $0,6 \pm 0,2^{\circ} \mathrm{C}$, e a década de 90 foi considerada a mais quente do último milênio (KERR, 2005; IPCC, 2007). Existem projeções de que a concentração do $\mathrm{CO}_{2}$ atmosférico duplicará até o final desse século, o que poderá provocar aumento na temperatura média do ar do planeta (STRECK, 2005; IPCC, 2007). Ainda não há unanimidade no valor do aumento da temperatura do ar global, devido ao aumento da concentração do $\mathrm{CO}_{2}$, mas estudos indicam um provável aumento entre 1,1 e $6,4^{\circ} \mathrm{C}$ na temperatura média do ar global até o final do século XXI, dependendo de cada região (MAHLMAN, 1997; IPCC, 2007).

Os efeitos da mudança climática sobre a produção das culturas podem ser complexos. O aumento da concentração de $\mathrm{CO}_{2}$ na atmosfera, em um primeiro momento, tende a ter um efeito positivo sobre as culturas, pois pode aumentar a taxa de crescimento das plantas, em razão do $\mathrm{CO}_{2}$ ser o substrato primário para fotossíntese. Por outro lado, se o aumento da concentração de $\mathrm{CO}_{2}$ for seguido de aumento da temperatura do ar, especialmente das temperaturas noturnas, poderá não haver aumento no crescimento e na produtividade das culturas, devido ao encurtamento do ciclo de desenvolvimento e ao aumento da respiração do tecido vegetal (SIQUEIRA et al., 2001; TAIZ \& ZEIGER, 2004; STRECK, 2005).

A atual concentração de $\mathrm{CO}_{2}$ no ambiente é insuficiente para saturar a enzima ribulose 1-5 bifosfato carboxilase-oxigenase (Rubisco), responsável pela carboxilação primária, que é o processo metabólico que governa a fotossíntese em plantas $\mathrm{C}_{3}$ (TAIZ \& ZEIGER, 2004). Consequentemente, com o aumento da concentração de $\mathrm{CO}_{2}$ atmosférico ocorre uma redução na fotorrespiração das plantas $\mathrm{C}_{3}$, por promover a carboxilação e diminuir a oxigenação da enzima Rubisco (SAGE, 1994). Já as plantas $C_{4}$ possuem uma enzima específica para o $\mathrm{CO}_{2}$, a enzima fosfoenolpiruvato carboxilase (PEP), que fixa o $\mathrm{CO}_{2}$ nas células do mesófilo e o transporta na forma de um ácido de quatro carbonos para as células da bainha vascular, onde ocorre o ciclo de Calvin, concentrando o $\mathrm{CO}_{2}$ nesse local. Portanto, as plantas $\mathrm{C}_{4}$ respondem menos ao aumento na concentração de $\mathrm{CO}_{2}$ do que as plantas $\mathrm{C}_{3}$, pois já possuem um mecanismo que aumenta a eficiência fotossintética na utilização de $\mathrm{CO}_{2}$ (TAIZ \& ZEIGER, 2004).

Dependendo da disponibilidade térmica em uma região e da cultura agrícola, altas temperaturas do ar podem levar à menor produtividade, em razão do aumento da taxa de desenvolvimento e do aumento da respiração. Porém, um ciclo de desenvolvimento mais curto pode ser benéfico, por exemplo, para que as fases críticas da cultura escapem de secas ou geadas. Além disso, o uso de cultivares de maturação tardia poderá compensar o efeito das altas taxas de desenvolvimento, em razão das temperaturas do ar mais elevadas (HIJMANS, 2003). Já em locais onde atualmente as temperaturas do ar baixas limitam a produção, o aquecimento global poderá contribuir para um alongamento da estação de crescimento e propiciar temperaturas do ar que venham a favorecer o crescimento das culturas (HIJMANS, 2003).

O objetivo desta revisão foi reunir informações da literatura sobre os possíveis efeitos do aumento da concentração de $\mathrm{CO}_{2}$ e da temperatura do ar no crescimento, no desenvolvimento e na produtividade da cultura da batata.

Efeitos do aumento da concentração de $\mathrm{CO}_{2}$ e da temperatura do ar sobre o crescimento das plantas de batata

A fixação de $\mathrm{CO}_{2}$ pelas plantas via fotossíntese converte a energia solar (radiação fotossinteticamente ativa - RFA) em energia química $\left(\mathrm{H}_{2} \mathrm{O}+\mathrm{CO}_{2}+\mathrm{RFA}=\left(\mathrm{CH}_{2} \mathrm{O}\right)+\mathrm{O}_{2}\right)$ que é utilizada para a manutenção e o crescimento dos vegetais (TAIZ \& ZEIGER, 2004). O aumento da concentração de $\mathrm{CO}_{2}$ 
promove um aumento da taxa fotossintética e uma diminuição da taxa de transpiração por unidade de área foliar e, consequentemente, uma melhor eficiência de uso da água, favorecendo uma maior produção de fotoassimilados (FLEISHER \& TIMLIN, 2006). No entanto, se houver o aumento da concentração de $\mathrm{CO}_{2}$ e da temperatura do ar, poderá haver a redução no crescimento das culturas (BUTTERFIELD \& MORISON, 1992), devido ao aumento da respiração (TAIZ \& ZEIGER, 2004), principalmente daquelas de metabolismo $\mathrm{C}_{3}$, como, por exemplo, a batata.

Experimentos realizados com batata indicam que o aumento da concentração de $\mathrm{CO}_{2}$ tem pouco efeito na produção de biomassa acima do solo, porém a biomassa abaixo do solo aumenta consideravelmente, devido ao aumento do número e tamanho dos tubérculos (CIB, 2008). Com o aumento da concentração de $\mathrm{CO}_{2}$, a biomassa alocada para os tubérculos é aumentada em detrimento das hastes, folhas e flores. Isso aumentou a fração da biomassa abaixo/acima do solo e diminuiu a porcentagem de nitrogênio nas folhas em 12,6\% (CONN \& COCHRAN, 2006). A elevada concentração de $\mathrm{CO}_{2}$ pode diminuir a estatura de planta, o número de folhas (HACOUR et al., 2002) e o conteúdo de clorofila, o que acelera a senescência (BINDI et al., 2002). Esse efeito do $\mathrm{CO}_{2}$ na redução do conteúdo da clorofila é considerado negativo e pode ser explicado pelos elevados níveis de $\mathrm{CO}_{2}$, que promovem acúmulo de amido nos cloroplastos (BINDI et al., 2002).

A cultura da batata é sensível a altas temperaturas do ar. A temperatura ótima para a fotossíntese é em torno de $24^{\circ} \mathrm{C}$, e a taxa fotossintética reduz rapidamente com a diminuição desta (WOLF et al., 1990; TIMLIN et al., 2006). Altas temperaturas do ar (acima da temperatura ótima) também resultam na redução da taxa fotossintética, possivelmente, devido à redução da eficiência do fotossistema II, ao aumento na respiração de manutenção e à redução da área foliar (PRANGE et al., 1990). LAFTA \& LORENZEN (1995) constataram que plantas de batata cultivadas em câmaras de crescimento sob temperaturas do ar de 31/ $29^{\circ} \mathrm{C}$ (dia/noite) apresentaram redução na produção de matéria seca de 44 a $72 \%$ depois de quatro semanas, em comparação com um regime de temperatura do ar de 19/17 C (dia/noite). Altas temperaturas do ar aumentam o período de crescimento das folhas, o que causa redução na taxa de translocação de carboidratos para os tubérculos (MARINUS \& BODLAENDER, 1975). Temperaturas do ar acima da ótima resultam em plantas de batata mais altas, com elevada massa seca de hastes e folhas menores (PRANGE et al., 1990; LAFTA \& LORENZEN, 1995), e reduzida produção de tubérculos em muitas cultivares (KHEDHER \& EWING, 1985).
Efeitos do aumento da concentração de $\mathrm{CO}_{2}$ e da temperatura sobre o desenvolvimento das plantas de batata

A concentração de $\mathrm{CO}_{2}$ atmosférico pode influenciar o desenvolvimento das culturas (CONN \& COCHRAN, 2006). Estudos realizados com quatro espécies anuais (Guara brachycarpa, Gailardia pulchella, Oenothera laciniata e Lupinus texensis) mostram que uma elevada concentração de $\mathrm{CO}_{2}$ pode levar ao aumento e à diminuição ou não ter efeito algum sobre o desenvolvimento das culturas (REEKIE \& BAZZAZ, 1991). MIGLIETTA et al. (1998) relataram que o florescimento e a senescência das folhas em batata foram antecipados quando a concentração de $\mathrm{CO}_{2}$ foi elevada. Similarmente, SCHAPENDONK et al. (2000) observaram que a senescência das folhas foi antecipada quando dobrada a concentração de $\mathrm{CO}_{2}$ em cultivares de ciclo tardio, porém não afetou a senescência das folhas em cultivares precoces de batata, em função do curto ciclo de desenvolvimento das últimas comparadas com as primeiras.

A temperatura do ar é o principal fator ambiental que afeta o desenvolvimento da cultura da batata (STRECK et al., 2007). Em geral, para que ocorra a máxima taxa de desenvolvimento, a temperatura do ar deve estar entre 14 e $22^{\circ} \mathrm{C}$ (MARINUS \& BODLAENDER, 1975). A temperatura média para a cultura da batata está entre 10 e $20^{\circ} \mathrm{C}$, e a faixa ideal para a indução e o crescimento dos tubérculos e a produção de matéria seca está entre 15 e $20^{\circ} \mathrm{C}$ (ANTUNES \& FORTES, 1981). No entanto, o efeito da temperatura do ar nas plantas de batata depende da fase de desenvolvimento (SILVA, 2004). Na fase vegetativa (emergência até o início da tuberização), a temperatura-base é de $4^{\circ} \mathrm{C}$, a temperatura ótima é de $15^{\circ} \mathrm{C}$ e a temperatura máxima é de $28^{\circ} \mathrm{C}$ (HARTZ \& MOORE, 1978; INGRAM \& McCLOUD, 1984) e, na fase de tuberização (início da tuberização até o início da senescência), a temperatura-base é de $7^{\circ} \mathrm{C}$, a temperatura ótima é de $21^{\circ} \mathrm{C}$ e a temperatura máxima é de $30^{\circ} \mathrm{C}$ (SANDS et al., 1979; MANRIQUE \& HODGES, 1989). Já para a fase da senescência (início da senescência até morte da planta) as temperaturas cardinais são as mesmas da fase vegetativa (STRECK et al., 2007).

Altas temperaturas, acima da temperatura ótima, favorecem o crescimento da parte aérea em detrimento dos tubérculos, o que afeta a quantidade e qualidade da produção, devido ao atraso no início da tuberização e à manifestação de defeitos fisiológicos e deformações nos tubérculos, como embonecamento, coração oco, rachaduras e necroses (MENEZES et al., 1999; VAN DAM et al., 1996; BISOGNIN \& STRECK, 
2009). A temperatura média ótima para o início da tuberização está entre 16 e $18^{\circ} \mathrm{C}$, sendo gradativamente menos favorável à medida que se aproxima dos limites de 6 e $29^{\circ} \mathrm{C}$ (BISOGNIN \& STRECK, 2009). MARINUS \& BODLAENDER (1975) estudaram oito cultivares de batata em três temperaturas $\left(16,22\right.$ e $\left.27^{\circ} \mathrm{C}\right)$, e um dos efeitos das altas temperaturas é a senescência mais rápida das folhas, com redução no enchimento de tubérculos pelo encurtamento do período de acúmulo de fotoassimilados. Temperatura do ar acima de $23^{\circ} \mathrm{C}$ retarda a fase de desenvolvimento da emergência ao início da tuberização devido ao aumento da fase vegetativa, o que ocasiona um atraso no início da tuberização (KOOMAN \& RABBINGE, 1996).

Simulando o desenvolvimento da cultura da batata com cenários de aumento da temperatura para Santa Maria, Rio Grande do Sul (RS), STRECK et al. (2006) encontraram aumentos na duração da fase vegetativa (emergência ao início da tuberização) e no ciclo total (emergência à colheita), com o aumento da temperatura do ar para o cultivo de outono (safrinha) e menor efeito no cultivo de primavera (safra), com acréscimo na duração da fase vegetativa e diminuição do ciclo total (STRECK et al., 2006). O aumento do ciclo total com o aumento da temperatura do ar projetada para o cultivo de outono ocorreu em razão da grande frequência de dias com temperaturas supraótimas durante a fase da emergência ao início da tuberização nessa época de cultivo (STRECK et al., 2006). Para fases intermediárias, as projeções dependem da fase e da época de cultivo. A fase do início da tuberização ao início da senescência, de acordo com os resultados das simulações, aumentará com o aumento da temperatura do ar no cultivo de outono e diminuirá no cultivo de primavera, enquanto que a fase do início da senescência a colheita terá menor duração em ambas as épocas de cultivo, mas no outono esta fase foi afetada apenas com aumentos de $6^{\circ} \mathrm{C}$ (Figura 1). Os efeitos das altas temperaturas na duração das fases de desenvolvimento da batata estão associados com a resposta da taxa de desenvolvimento à temperatura do ar (STRECK et al., 2007).

Alterações na duração das fases de desenvolvimento da batata poderão ter consequências no manejo da cultura, por exemplo, se houver um aumento na duração do ciclo total devido ao aumento da temperatura no cultivo de outono em regiões subtropicais, poderá ter impacto negativo na qualidade dos tubérculos, pois a colheita sendo atrasada nesses locais ocorreria em condições meteorológicas adversas, no inverno, quando a umidade do solo é elevada (STRECK et al., 2006). Além disso, tratos culturais são afetados, sendo alguns adiantados e outros atrasados em consequência das alterações das fases de desenvolvimento. As consequências no manejo da cultura podem ser minimizadas pelo melhoramento genético por meio do desenvolvimento de cultivares adaptadas a altas temperaturas.

Efeitos do aumento da concentração de $\mathrm{CO}_{2}$ e da temperatura do ar sobre a produtividade da batata

A batata responde ao aumento da concentração de $\mathrm{CO}_{2}$ com aumento da biomassa total e produtividade. SCHAPENDONK et al. (2000) e SICHER \& BUNCE (1999) relatam aumento de 27 a $49 \%$ na produtividade de tubérculos quando foi dobrada a concentração de $\mathrm{CO}_{2}(740$ ppmv). Porém, com a elevação da temperatura do ar, o aumento na produtividade pelo aumento de $\mathrm{CO}_{2}$ pode não se confirmar, devido principalmente ao encurtamento do ciclo e aumento da respiração vegetal (BUTTERFIELD \& MORISON, 1992; SIQUEIRA et al., 2001). As temperaturas noturnas são as mais determinantes para a cultura da batata, pois interferem diretamente na tuberização e no enchimento de tubérculos. Temperaturas noturnas acima de $20^{\circ} \mathrm{C}$ inibem a tuberização (SLATER, 1968), e temperaturas mínimas entre 8 e $15^{\circ} \mathrm{C}$, associadas a amplitudes térmicas entre 10 e $15^{\circ} \mathrm{C}$, são as mais favoráveis ao acúmulo de matéria seca nos tubérculos (BURTON, 1981).

Em nível mundial, há uma grande preocupação com os efeitos da mudança climática na cultura da batata. Estudos indicam que o aumento na temperatura do ar poderá reduzir a produção de batata entre 10 e $19 \%$ até o ano 2039 e entre 18 e 32\% entre os anos de 2040 e 2069, e a zona tropical seria a região mais vulnerável, onde a perda pode superar os $50 \%$ (HIJMANS, 2003). Outros estudos indicam um possível aumento da produtividade na Inglaterra (DAVIES et al., 1996), Escócia (PEIRIS et al., 1996) e Finlândia (CARTER et al., 1996). As regiões onde o aquecimento global terá um efeito benéfico na produtividade de tubérculos de batata são, principalmente, regiões de maior latitude e de clima temperado, como Canadá e Rússia (HIJMANS, 2003). Entretanto, a maioria das pesquisas indica uma redução na produtividade de tubérculos de batata em altas temperaturas. Estudos realizados em Lavras, Minas Gerais (MG), constataram atraso no inicio da tuberização e redução na partição dos fotoassimilados para os tubérculos em razão das altas temperaturas, o que reduziu a produtividade da batata em 25,5\% (MENEZES etal., 1999). ROSENZWEIG et al. (1996), nos Estados Unidos, relataram uma redução na produtividade de tubérculos de batata de 1,4\%, 3,4\% e $18,5 \%$ em aumentos de temperatura de $1,5^{\circ} \mathrm{C}, 2,5^{\circ} \mathrm{C}$ e $5,0^{\circ} \mathrm{C}$, respectivamente. Altas temperaturas, em regiões 


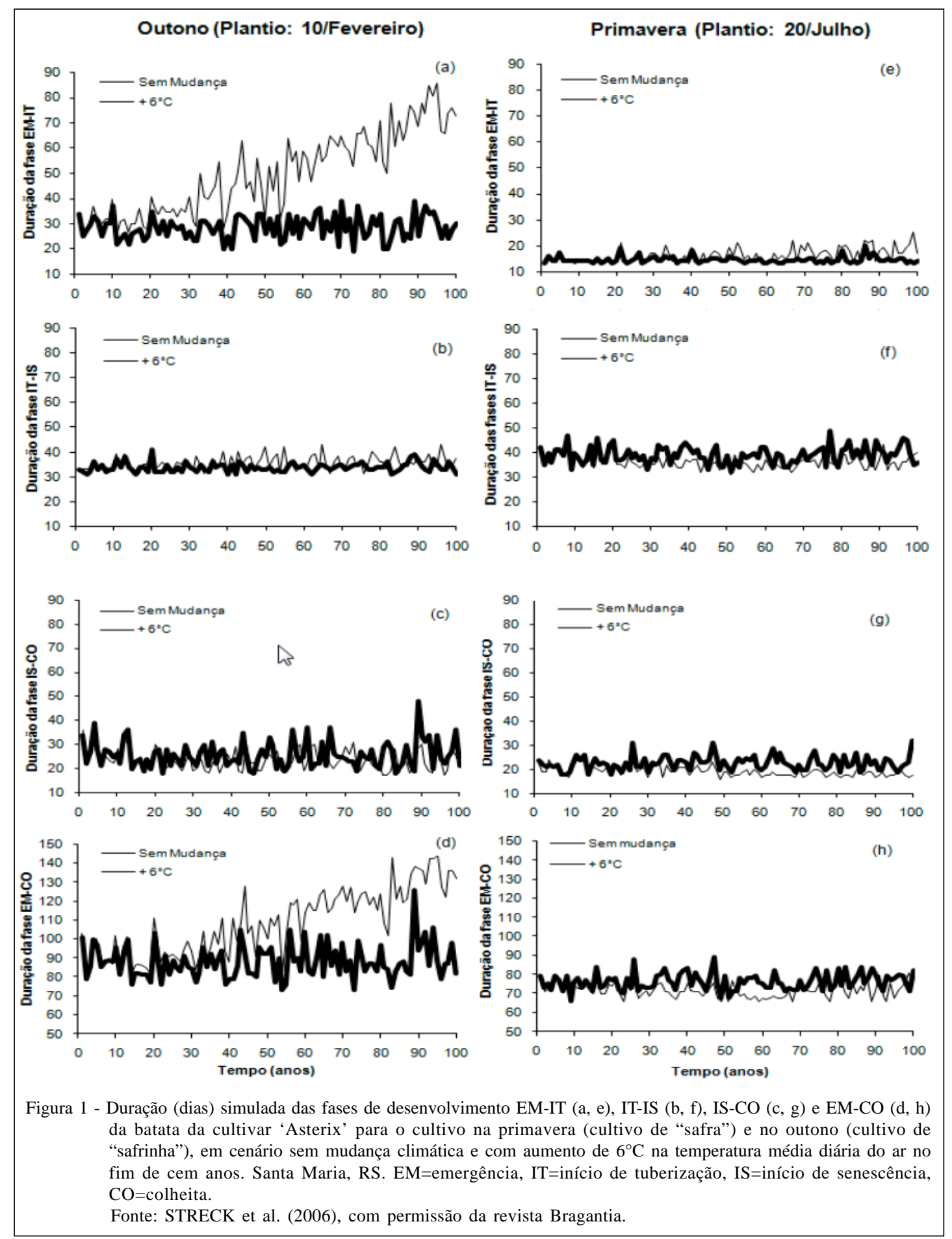

de clima subtropical, encurtam a dormência e aceleram o envelhecimento fisiológico dos tubérculos, reduzindo o tempo de armazenamento. Altas temperaturas aceleram a duração da fase de tuberização, dependendo da cultivar e da época de plantio, e nas condições de cultivo do Brasil quanto menor a fase de tuberização, menor a produtividade de tubérculos (BISOGNIN \& STRECK, 2009). Nessa fase, a temperatura do ar tem um grande efeito sobre a produtividade de tubérculos, pois a cada aumento de $1^{\circ} \mathrm{C}$ da temperatura entre 15 e $25^{\circ} \mathrm{C}$ ocorre uma redução média da produtividade de $1 \%$. Além disso, a produtividade dos tubérculos na temperatura de $30^{\circ} \mathrm{C}$ 
é a metade daquela de $20^{\circ} \mathrm{C}$ e ainda menor do que a de $10^{\circ} \mathrm{C}$ (BISOGNIN \& STRECK, 2009).

Uma possível alternativa de manejo para diminuir a exposição às altas temperaturas durante o crescimento dos tubérculos e obter uma duração mais longa da área foliar seria antecipar a data de plantio (HIJMANS, 2003), pois essa prática diminui os efeitos negativos das altas temperaturas na produtividade dos tubérculos de batata (ROSENZWEIG et al., 1996). Estimativas indicam que antecipar o plantio, sobretudo em regiões áridas, poderia reduzir pela metade a diminuição prevista na produção mundial de batata. No Sul da Europa, o plantio antecipado aumenta a produção de batata e reduz as necessidades de água, tanto na situação atual, quanto nas projeções para o futuro. Porém, na prática, a adaptação pode não ser tão fácil, em função da disponibilidade de água, das pragas e doenças e do mercado consumidor (FOCUS, 2008). Outra estratégia seria a expansão para novas áreas, para zonas mais produtivas ou para aquelas em que, atualmente, não se produz batata e permitam o seu cultivo, como em algumas zonas tropicais de grande altitude. Em algumas regiões na China, por exemplo, espera-se que a batata se torne uma possível cultura de inverno (HIJMANS, 2003). Em último caso, dependendo da vulnerabilidade da região às novas disponibilidades climáticas, talvez terá que se optar pelo abandono da produção de batata (FOCUS, 2008; HALL \& ZISKA, 2000).

Outra possível alternativa seria utilizar cultivares de ciclo tardio para manter a área foliar verde por um tempo mais longo, o que resulta em aumento de produtividade (HIJMANS, 2003). No entanto, essas alternativas são aplicáveis em regiões de clima temperado, pois, em regiões de clima subtropical, a temperatura do ar e o fotoperíodo diminuem durante o ciclo das plantas no cultivo de outono, enquanto que a disponibilidade desses elementos climáticos aumenta com o avanço do ciclo das plantas durante o cultivo de primavera (BISOGNIN et al., 2008; BISOGNIN \& STRECK, 2009). Durante o cultivo de outono, além da menor disponibilidade de radiação solar, as plantas também apresentam uma menor eficiência de sua utilização, pela menor área foliar que intercepta a radiação incidente (BISOGNIN et al., 2008). O cultivo de primavera em condições de clima subtropical favorece o crescimento de parte aérea e tubérculos, ou seja, o potencial produtivo é maior, em razão da maior disponibilidade de radiação e amplitude térmica (BSOGNIN \& STRECK, 2009).

A busca de tolerância às altas temperaturas deve ser importante para a sustentabilidade da cadeia produtiva brasileira de batata no futuro. Estudo nesse sentido foi realizado em Minas Gerais por TEIXEIRA et al. (2007), com avaliação de clones de batata tolerantes ao calor, tendo sido identificados 20 dos 91 clones testados com potencial para o cultivo em regiões de altas temperaturas, com alta produtividade e alto teor de matéria seca nos tubérculos, indicando que a batata tem variabilidade para alta temperatura.

Efeitos indiretos por doenças do aumento da concentração de $\mathrm{CO}_{2}$ e da temperatura do ar sobre a cultura da batata

Efeitos indiretos da mudança climática sobre a produtividade de batata relacionados a doenças são importantes para a cadeia produtiva da batata, tendo em vista as novas técnicas ou métodos de controle das doenças (CHAKRABORTY \& PANGGA, 2004; GHINI, 2005). Por meio da modelagem matemática, é possível fazer simulações em cenários de mudança climática de doenças em diferentes níveis de severidade, com o objetivo de estimar a produtividade e estabelecer estratégias e métodos de controle (GHINI et al., 2008). Assim, LOPES et al. (2008), considerando as previsões de aumento na temperatura global, informam que, na época de cultivo das chuvas, a cultura de batata será mais atingida com doenças do que na época de cultivo de inverno em regiões tropicais, e a colheita não será viável em regiões de baixa altitude, devido ao aumento de doenças.

Segundo HAVERKORT \& VERHAGEN (2008), os problemas com as doenças devem aumentar em razão do aumento das temperaturas durante a estação de crescimento da cultura, e o controle da requeima da batata (Phytophthora infestans) será mais difícil, devido a chuvas mais intensas e de mais curta duração projetadas para o futuro. CARTER et al. (1996) concluíram que o aquecimento global deve expandir a área de cultivo em 2050, o que fará com que o risco de doenças como o cisto da batata (Globodera rostochiensis) e a requeima da batata seja significativamente maior na Finlândia, região de clima temperado.

Em geral, o aumento da temperatura do ar favorece a incidência de doenças como a murchadeira (Ralstonia solanacearum) e a podridão mole (Pectobacterium spp.), mas a requeima da batata, doença bastante preocupante para os produtores, pode tornar-se menos agressiva, pois a requeima é favorecida por baixas temperaturas (ABBA, 2009b).

\section{CONCLUSÃO}

O aumento do $\mathrm{CO}_{2}$, seguido de aumento na temperatura do ar, de maneira geral, resultará em menor 
crescimento, redução na duração do ciclo de desenvolvimento, menor produtividade e aumento da incidência de doenças da batata. Como estratégia para minimizar os efeitos de um possível aquecimento global sobre essa cultura, sugere-se que sejam desenvolvidas cultivares tolerantes a altas temperaturas, adaptadas as épocas de plantio em cada local, alteradas as práticas de manejo da cultura e até expandidas as áreas de cultivo para regiões mais frias.

\section{REFERÊNCIAS}

ABBA. Associação Brasileira de Batata. A batata como alimento. Disponível em: <http://www.abbabatatabrasileira.com.br>. Acesso em: 15 jun. 2009a.

ABBA. Associação Brasileira de Batata. A batata e o aquecimento global. Disponível em: <http://www.abbabatatabrasileira.com.br>. Acesso em: 15 jun. 2009b.

ANTUNES, F.Z.; FORTES, M. Exigências climáticas da cultura da batata. Informe Agropecuário, v.7, n.76, p.19-23, 1981.

BINDI, M. et al. Chlorophyll concentration of potatoes grown under elevated carbon dioxide and/or ozone concentrations. European Journal of Agronomy, v.17, p.319-335, 2002. Disponível em: $<$ http://cat.inist.fr/?aModele=afficheN\&cpsidt=13968416>. Acesso em: 17 out. 2009. doi:10.1016/S1161-0301(02)00069-2.

BISOGNIN, D.A. et al. Desenvolvimento e rendimento de clones de batata na primavera e no outono. Pesquisa Agropecuária Brasileira, v.43, n.6, p.699-705, 2008. Disponível em: <http://www.scielo.br/pdf/pab/v43n6/ a05v43n6.pdf>. Acesso em: 17 out. 2009. doi: 10.1590/S0100204X2008000600005.

BISOGNIN, D.A.; STRECK, N.A. Desenvolvimento e manejo das plantas para alta produtividade e qualidade da batata. . Itapetininga: Associação Brasileira da Batata, 2009. 30p.

BURTON, W.G. Challenges for stress physiology in potato. American Potato Journal, v.58, n.1, p.3-14, 1981.

BUTTERFIELD, R.E.; MORISON, J.I.L. Modeling the impact of climate warming on winter cereal development. Agricultural and Forest Meteorology, v.62, n.3, p.241261, 1992.

CARTER T.R. et al. Assessing the risks and uncertainties of regional crop potential under a changing climate in Finland. Agricultural and Food Science, v.5, p.329-350, 1996. Disponível em: <www.ingentaconnect.com/content/mtt/afsf $>$. Acesso em: 18 out. 2009. doi: 10.1016/0168-1923(95)02287-2.

CHAKRABORTY, S.; PANGGA, I.B. Plant disease and climate change. In: ILlingS, M.; HOLMES, A. (Ed.). Plant microbiology. London: BIOS Scientific, 2004. p.163-180.

CIB. Centro de Inteligência da Batata. Informativo quinzenal da batata. Lavras, 2008. 8p. (Informativo n.30, 11 de setembro de 2008).
CONN, J.S.; COCHRAN, V.L. Response of potato (Solanum tuberosum L.) to elevated atmospheric $\mathrm{CO}_{2}$ in the North American Subarctic. Agriculture, Ecosystems and Environment, v.112, p.49-57, 2006. Disponível em: <http:/ /www3.interscience.wiley.com/journal/119164188>. Acesso em: 16 jul. 2009. doi: 10.1016/j.agee.2005.07.010.

DAVIES A. et al. Modelling the predicted geographic and economic response of UK cropping systems to climate change scenarios: the case of potatoes. Aspects of Applied Biology, v.45, p.63-69, 1996. Disponível em: <http://cat.inist.fr/ ?aModele $=$ afficheN\&cpsidt $=17508082>$. Acesso em: 16 jul. 2009. doi: 10.1111/j.1744-7348.1997.tb05792.x.

FAOSTAT. The agricultural production domain covers. Disponível em: <http://www.fao.org/corp/statistics.html>. Acesso em: 15 jun. 2009.

FLEISHER, D.H.; TIMLIN, D. Modeling expansion of individual leaves in the potato canopy. Agricultural and Forest Meteorology, v.139, p.84-93, 2006. Disponível em: <http:// w w w. ars.us d a.gov/s p 2 U s e r Files/ad_ho c/ 12755100FullTextPublicationspdf/Publications/fleisher/ Modeling_Expansion_of_Individual_Leaves_in_the_Potato_Canopy.pdf $>$. Acesso em: 20 set. 2009. doi: 10.1016/j.agrformet.2006.06.002.

FOCUS. Potato and climate change, 2008. 16p. (InfoResources Focus No 1/08). Disponível em: <www.potato2008.org/en/ index.html>. Acesso em: 15 jun. 2009.

GHINI, R. Mudanças climáticas globais e doenças de plantas. Jaguariúna: Embrapa Meio Ambiente, 2005. 104p.

GHINI, R. et al. Risk analysis of climate change on coffee nematodes and leaf miner in Brazil. Pesquisa Agropecuária Brasileira, v.43, p.187-194, 2008.

HACOUR, A. et al. $\mathrm{CO}_{2}$ and ozone effects on canopy development of potato crops across Europe. European Journal of Agronomy, v.17, p.257-272, 2002. Disponível em: <http://www.sciencedirect.com/ science?_ob=ArticleURL\&_udi=B6T67-46YXF0M$5 \&$ \&_user $=10 \&$ \&_doc $=1 \&$ \& fmt $=\&$ \&_orig $=$ search \&_sort $=\mathrm{d}$ $\&$ docanchor $=\&$ view $=$ c $\&$ _searchStrId $=110264260$ $8 \&$ \&_rerun Orig in $=$ g o o g l e \&_a c c t $=$ C 0000 $50221 \&$ \& version $=1 \&$ \&_url Version $=0 \&$ \& userid $=$ $10 \& m d 5=3 f$ c741c4835729a8cfdaea972d1854e2>. Acesso em: 15 jun. 2009. doi: 10.1016/S1161-0301(02)00065-5.

HALL, A.E.; ZISKA, L.H. Crop breeding strategies for the 21st century. In: REDDY, K.R.; HODGES, H.F. Climate change and global crop productivity. London: British Library, 2000. p.407-419.

HARTZ, T.K.; MOORE, F.D. Prediction of potato yield using temperature and insolation data. American Potato Journal, v.55, n.7, p.431-436, 1978.

HAVERKORT, A.J.; VERHAGEN, A. Climate change and its repercussions for the potato supply chain. Potato Research, v.51, n.3-4, p.223-237, 2008.

HIJMANS, R.J. The Effect of climate change on global potato production. American Journal of Potato Research, v.80, p.271-280, 2003. Disponível em: <http://www.irri.org/gis/docs/ 2003-Hijmans-Climate_change_potato.pdf>. Acesso em: 23 abr. 2009. doi: 10.1007/BF02855363. 
INGRAM, K.T.; McCLOUD, D.E. Simulation of potato growth and development. Crop Science, v.24, n.1, p.21-27, 1984.

IPCC [Intergovernmental Panel on Climate Change]. Climate change 2007: The physical Science Basis - Contribution of Working Group I to the Fourth assessment report of the Intergovernmental Panel on Climate Change. Cambridge: University, 2007. 989p. Disponível em: <http:// www.ipcc.ch/ipccreports/ar4-wg1.htm>. Acesso em: 11 maio, 2009.

KERR, R.A. Millennium's hottest decade retains its title, for now. Science, v.307, p.828-829, 2005.

KHEDHER, M.B.; EWING, E.E. Growth analysis of eleven potato cultivars grown in the greenhouse under long photoperiods with and without heat stress. American Potato Journal, v.62, n.10, p.537-554, 1985. Disponível em: <http:/ /www.springerlink.com/content/5032t1m8r7715340/>. Acesso em: 23 abr. 2009. doi: 10.1007/BF02854401.

KOOMAN, P.L.; RABBINGE, R. An analysis of the relation between dry matter allocation to the tuber and earliness of a potato crop. Annals of Botany, v.77, n.3, p.235-242, 1996. Disponível em: <http://aob.oxfordjournals.org/cgi/reprint/77/ 3/235>. Acesso em: 11 maio, 2009. doi:10.1006/ anbo.1996.0027.

LAFTA, A.M.; LORENZEN, J.H. Effect of high temperature on plant growth and carbohydrate metabolism in potato. Plant Physiology, v.109, p.637-643, 1995. Disponível em: <http:/ /www.ncbi.nlm.nih.gov/pmc/articles/PMC157630/pdf/ 1090637.pdf>. Acesso em: 11 maio, 2009.

LOPES, C.A. et al. Impacto potencial das mudanças climáticas sobre as doenças da batata no Brasil. In: GHINI, R.; HAMADA, E. Mudanças climáticas: impactos sobre doenças de plantas no Brasil. Brasília: Embrapa/SCT, 2008. Cap.3, p.75-92.

MAHLMAN, J.D. Uncertainties in projections of human induced climate warmings. Science, v.278, n.5342, p.14161417, 1997.

MANRIQUE, L.A.; HODGES, T. Estimation of tuber initiation in potatoes grown in tropical environments based on different methods of computing thermal time. American Potato Journal, v.66, n.7, p.425-436, 1989

MARINUS, J.; BODLAENDER, K.B.A. Response of some potato varieties to temperature. Potato Research, v.18, n.2, p.189201, 1975. Disponível em: <http://www.springerlink.com/content/ a103j1807m11n848>. Acesso em: 11 maio, 2009. doi: 10.1007/ BF02361722

MEARNS, L.O. Climate change and variability. In REDDY, K.R.; HODGES, H.F. Climate change and global crop productivity. London: British Library, 2000. p.7-35. Disponível em: <http://books.google.com.br/ books? id=L 26c ZB9lVqYC\&dq=Climate +change + an $\mathrm{d}+$ global+crop+productivity\&printsec $=$ frontcover\&source=bn\&hl= p t B R \& e i = q P n c S v y K N M 29 l A e b 2 b m h A $\mathrm{Q} \& \mathrm{sa}=\mathrm{X} \&$ o $\mathrm{i}=\mathrm{b}$ oo ok_result \& ct $=$ result \& resnum $=4$ \&ved $=0$ CBwQ6AEwAw\#v $=$ onepage $\& \mathrm{q}=\& \mathrm{f}=$ false $>$. Acesso em: 24 jun. 2009.
MENEZES, C.B. et al. Avaliação de genótipos de batata (Solanum tuberosum L.) nas safras das águas e inverno no sul de Minas Gerais. Ciência e Agrotecnologia, v.23, n.4, p.777784, 1999. Disponível em: <http://www.editora.ufla.br/revista/ 23_4/art02.pdf $>$. Acesso em: 24 jun. 2009.

MIGLIETTA, F. et al. Free air $\mathrm{CO}_{2}$ enrichment of potato (Solanum tuberosum L.): development growth and yield. Global Change Biology, v.4, p.163-172, 1998.

PRANGE, R.K. et al. Reduction in potato growth at high temperature: Role of photosynthesis and dark respiration. American Potato Journal, v.67, p.357-369, 1990. Disponível em: <http://www.springerlink.com/content/ b78356770941xj43>. Acesso em: 11 maio, 2009. doi: 10.1007/ BF02987277.

PEIRIS, D.R. et al. A simulation study of crop growth and development under climate change. Agricultural and Forest Meteorology, v.79, p.271-287, 1996. Disponível em: <http:/ /cat.inist.fr/?aModele=afficheN\&cpsidt $=3140760>$. Acesso em: 11 maio, 2009. doi: 10.1016/0168-1923(95)02286-4.

REEKIE, E.G.; BAZZAZ, F.A. Phenology and growth in four annual species grown in ambient and elevated $\mathrm{CO}_{2}$. Canadian Journal of Botany, v.69, p.2475-2481, 1991.

ROSENZWEIG, C.J. Potential impacts of climate change on citrus and potato production in the US. Agricultural Systems, v.52, n.4, p.455-479, 1996. Disponível em:<http:// www.ingentaconnect.com/content/els/0308521x/1996/ 00000052/00000004/art00059>. Acesso em: 14 jul. 2009. doi: 10.1016/0308-521X(95)00059-E.

SAGE, R.F. Acclimation of photosynthesis to increasing atmospheric $\mathrm{CO}_{2}$. The gas exchange perspective. Photosynthesis Research, v.39, p.351-368, 1994. Disponível em: <http:// www.springerlink.com/content/g86655j665860879>. Acesso em: 19 ago. 2009. doi: 10.1007/BF00014591.

SANDS, P.J. et al. A model of the development and bulking of potatoes (Solanum tuberosum L.). I. Derivation from wellmanaged field crops. Field Crops Research, v.2, n.4, p.309311, 1979.

SCHAPENDONK, H.C.M. et al. Effects of elevated $\mathrm{CO}_{2}$ concentration on photosynthetic acclimation and productivity of two potato cultivars grown in open-top chambers. Australian Journal of Plant Physiology, v.27, p.1119-1130, 2000. Disponível em: <http:// cat.inist.fr/?aModele=afficheN\&cpsidt=819851 >. Acesso em: 24 set. 2009 .

SICHER, R.C.; BUNCE, J.A. Photosynthetic enhancement and conductance to water vapor of field-grown Solanum tuberosum (L.) in response to $\mathrm{CO}_{2}$ enrichment. Photosynthesis Research, v.62, p.155-163, 1999. Disponível em: <http:// www.springerlink.com/content/t31551447mm1h040>. Acesso em: 24 set. 2009. doi: 10.1023/A:1006327931675.

SILVA, L.A.S. Duração do ciclo vegetativo e sua relação com o potencial produtivo de genótipos de batata. 2004 106f. Dissertação (Mestrado em genética e melhoramento de plantas) - Universidade Federal de Lavras, Lavras, MG.

SIQUEIRA, O.J.W. et al. Efeitos potenciais das mudanças climáticas na agricultura brasileira e estratégias adaptativas para 
algumas culturas. In: LIMA, M.A. et al. Mudanças climáticas globais e a agropecuária brasileira. Jaguariúna: Embrapa, 2001. p.33-63.

SLATER, J.W. The effect of night temperature on tuber initiation of the potato. Potato Research, v.11, n.1, p.1422, 1968.

STRECK, N.A. Climate change and agroecosystems: the effect of elevated atmospheric $\mathrm{CO}_{2}$ and temperature on crop growth, development, and yield. Ciência Rural, v.35, n.3, p.730740, 2005. Disponível em: <http://www.scielo.br/pdf/cr/v35n3/ a41v35n3.pdf>. Acesso em: 24 jun. 2009. doi: 10.1590/S010384782005000300041.

STRECK, N.A. et al. Simulação do desenvolvimento da batata cultivar Asterix em cinco cenários de mudanças climáticas em Santa Maria, RS. Bragantia, v.65, n.4, p.693-702, 2006. Disponível em: <http://www.scielo.br/pdf/brag/v65n4/21.pdf>. Acesso em: 24 jun. 2009. doi: 10.1590/S000687052006000400021 .
STRECK, N.A. et al. Simulating the development of field grown potato (Solanum tuberosum L.). Agricultural and Forest Meteorology, v.142, n.1, p.1-11, 2007.

TAIZ, L.; ZEIGER, E. Fisiologia vegetal. 3.ed. Porto Alegre: Artemed, 2004. 719p.

TIMLIN, D. et al. Whole plant photosynthesis, development, and carbon partitioning in potato as a function of temperature. Agronomy Journal, v.98, p.1195-1203, 2006.

TEIXEIRA, A.L. et al. Avaliação de clones de batata tolerantes ao calor. Batata Show, n.19, p.1-5, 2007. Disponível em: <http://www.abbabatatabrasileira.com.br/revista19_026.htm>. Acesso em: 24 jun. 2009.

VAN DAM, J. et al. Effects of temperature and photoperiod on early growth and final number of tuber in potato (Solanum tuberosum L.). Potato Research, v.39, n.1, p.51-62, 1996. Disponível em: <http://www.springerlink.com/content/ 05854087674q46t1>. Acesso em: 24 set. 2009. doi: 10.1007/ BF02358206.

WOLF, S. et al. Effect of high temperature on photosynthesis in potatoes. Annals of Botany, v.65, p.179-185, 1990. 